\title{
Density-based Food Analysis Using a Smartphone
}

\author{
Stephanie Knowlton ${ }^{1}$, Savas Tasoglu $\mathbf{u}^{1,2,3,4, *}$ \\ ${ }^{1}$ Department of Biomedical Engineering, University of Connecticut, Storrs, CT 06269. \\ ${ }^{2}$ Department of Mechanical Engineering, University of Connecticut, Storrs, CT 06269. \\ ${ }^{3}$ Institute of Materials Science, University of Connecticut, Storrs, CT 06269. \\ ${ }^{4}$ Institute for Collaboration on Health, Intervention, and Policy, University of Connecticut, Storrs, CT 06269. \\ *savas.tasoglu@uconn.edu
}

Received: 13 December 2018

Accepted: 13 May 2019

DOI: $10.18466 /$ cbayarfbe.496371

\begin{abstract}
Density is a crucial factor of interest in the food industry because it can reveal valuable information about the content and quality of food products. Traditionally, this physical property is quantified using a hydrometer or a pyncometer. However, the accuracy of analog instruments is limited by human error and more modern digital adaptations of such methods come with high monetary costs. Here, we present a lowcost, portable, and user-friendly platform for density-based analysis of liquid food samples of very small volumes $(<10 \mu \mathrm{L})$ via magnetic levitation. The platform is fully compatible with an Android smartphone which collects magnified images and conducts automated density-based metric determination using a custom-designed Android application. Validity of the device was shown by measuring the density of oils (indicating fat content) and ethanol solutions (indicating alcohol content). This technique offers an accurate and low-cost alternative to current density measurement techniques for analysis of food quality for broad use in-home or in the food industry.
\end{abstract}

Keywords: Magnetic levitation; magnetophoresis, food analysis, maglev, smartphone.

\section{Introduction}

According to the World Food Programme, there exists a need for standard operating procedures to analyse food quality and safety throughout the supply chain. There is also a push to develop stronger monitoring and data collection systems for tracking food safety and quality [1]. This call for action necessitates further research into methods for rapid and precise analysis of food and liquids. Density is a physical property of a sample and is defined as the ratio of mass to volume. Density determination (densiometry) is a common and valuable tool for analyzing food, primarily of liquids but also of solid products [2].

Density of liquids is most commonly determined using a pyncometer to measure the weight of a fixed volume of a sample of the liquid within a vessel and calibrating to the weight of pure water which is held in the same vessel [2]. Density (D) can be determined by the equation $D_{\text {water }} / M_{\text {water }}=D_{\text {unknown }} / M_{\text {unknown }}$, where $M$ is the mass of the liquid in the vessel. A hydrometer as an alternative instrument based on the principle that a floating object will displace the same weight of any liquid in which it floats, allowing for the density of any liquid (D) to be determined relative to that of water according to $\mathrm{V}_{\text {water }} \mathrm{D}_{\text {water }}=\mathrm{V}_{\text {unknown }} \mathrm{D}_{\text {unknown, }}$, where $\mathrm{V}$ represents volume displaced [2]. In the case of a pyncometer or a hydrometer, the accuracy and precision of these measurements depends fully upon the accuracy and precision of the mass and volume measurements, respectively.

One device currently on the market is a portable density meter (Anton Paar). This instrument records the density or concentration of a solution with an accuracy of 0.001 $\mathrm{g} / \mathrm{cm}^{3}$ and repeatability of $0.0005 \mathrm{~g} / \mathrm{cm}^{3}$. Though relatively portable, the device weighs $345 \mathrm{~g}$, requires a sample size of $2 \mathrm{~mL}$ (which may represent a significant loss in some settings, such as pharmaceuticals), and is marketed at a high cost (greater than \$2,500) [3].

Magnetic levitation is a method which levitates an object in a magnetic field based its density and magnetic susceptibility relative to those of the medium [4, 5]. When a diamagnetic sample is suspended in a paramagnetic solution, it experiences a buoyancy force and a magnetic force. The buoyancy force due to gravity depends on its density relative to that of the medium (the force acts upward if the sample is less dense than the medium and downward if it is denser than the medium). A magnetic force is created using a magnetic field is created by two magnets placed with like poles arranged facing each other along an axis parallel to the gravitational force. Within this magnetic field, a relatively diamagnetic object in a paramagnetic solution will experience magnetic forces acting on it in the direction of the centerline between the two magnets. The magnitude of this force is greatest near the surface of the 
magnets and decreasing gradually toward the centerline. Thus, depending on the relative density and magnetic susceptibility of a sample, it will levitate at a location within the magnetic field at which the buoyancy force is equal and opposite of the magnetic force (Supplementary Figure 1).

Magnetic levitation has previously been proposed by Mirica, et al. for the determination of density of solids and liquids based on their levitation height in a magnetic field when suspended in a paramagnetic solution [6]. In this study, the density of vegetable oils, milk, cheese, peanut butter, and various grains was determined in order to determine the fat content and the density of salt water solutions was measured to estimate salinity. Other applications of density assessment using magnetic levitation include analysis of forensic evidence [7], bottom-up tissue engineering [8, 9], polymer composition [10], chemical reaction progress over time, degree of protein binding [11,12], and degree of antigen binding [13].

Density can be indicative of the concentration of a solution (such as the sugar content in water), the index of solids in a liquid (such as in milk), or the length and degree of unsaturation of glyceride fatty acids. One application includes a lactometer to measure the milk solid content or water adulteration of milk on a scale of $25-35$, corresponding to densities of 1.025 to $1.035 \mathrm{~g} / \mathrm{mL}$. Salometers determine the salt content of water brines and, similarly, saccarometers measure the sugar content in sugar solutions [2]. Previously, magnetic levitation has been demonstrated as a viable method for measuring the salinity of a salt brine. Using a similar method to measure the concentration of a solute in a solution, an alcoholmeter is used determine the alcohol content by volume on a Tralle scale (from 0 to $100 \%$ ) or on a proof scale (from 100 under proof to 100 over proof), where $50 \%$ on the Tralle scale is equivalent to 0 on the proof scale [2]. These instruments are commonly used in production of alcoholic beverages to monitor the alcohol content during distillation [12]. Another application is an oleometer, which measures the density of oils on a scale of $50-0^{\circ}$, which corresponds to densities of $0.870-0.897$ $\mathrm{g} / \mathrm{mL}$ [2]. Saturated fatty acids are fatty acids in which the hydrocarbon chains are saturated with hydrogen and carbon-carbon bonds are single bonds only. In contrast, saturated fatty acids are fatty acids which contain double bonds between carbons; monounsaturated fatty acids contain only one double bond while polyunsaturated fatty acids contain more than one. Previous work has demonstrated that magnetic levitation can be used to determine the degree of unsaturation of oils (the ratio of unsaturated:saturated fatty acid content) [6].

Our group has previously developed portable, low-cost, and user-friendly alternative to this platform which is compatible with a Samsung Galaxy S4 smartphone and is capable of quantitative density determination of micron-scale samples (10-100 $\mu \mathrm{m}$ diameter) [14]. We have demonstrated its clinical applicability on diagnosis of sickle cell disease [14] and fluorescent cell imaging [15]. Here, we used a similar approach to analyze liquid droplets of food products. We demonstrate applicability of this platform for performing the density determination functions of both an oleometer to indirectly measure the fat contents of several types of oil and of an alcoholmeter to indirectly measure the alcohol content of a dilution series of ethanol-water solutions. The compact size, light weight, and small magnets relative to previous magnetic levitation designs demonstrates superior portability, affordability, and wide applicability of the setup. Previous magnetic levitation platforms [9] used relatively large $5 \mathrm{~cm}$ by $5 \mathrm{~cm}$ by $2.5 \mathrm{~cm}$ magnets; this can interfere with electronic devices in close proximity, limiting the portability of such magnetic levitation setups. The platform presented here is very low cost $(<\$ 100$ to fabricate) than the commercial densiometry products discussed previously. The novel platform presented here offers a rapid analysis of food and liquid for on-site quality control and for in-home food quality determination of oil and alcoholic beverages.

\section{Materials and Methods \\ 2.1. Oil Samples}

Oil samples were levitated in $0.1 \mathrm{mM}$ gadolinium chloride dissolved in 62:38 methanol:water solution. 200 $\mu \mathrm{L}$ of oil was suspended in $1 \mathrm{~mL}$ of paramagnetic solution by pipetting the oil into the solution then vortexing for 20 seconds to obtain droplets small enough to be inserted into the microcapillary but large enough to be focused and imaged. Table 1 shows the range of oil samples tested with the nutrition facts listed. Coconut oil was solid at room temperature, so the sample was melted gently prior to droplet formation then used as described. Levitation was imaged within 5 minutes of mixing the sample with the paramagnetic solution in order to prevent droplet aggregation and adhesion to the capillary walls.

Table 1. Oil varieties tested with reported oil content and levitation height.

\begin{tabular}{|c|c|c|c|c|c|c|}
\hline & $\begin{array}{l}: \tilde{\Xi} \\
\vdots \\
\vdots \\
\vdots \\
0\end{array}$ & 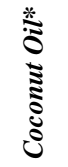 & 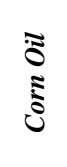 & 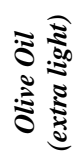 & 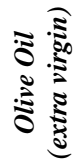 & 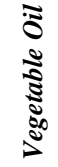 \\
\hline \multirow{5}{*}{$\begin{array}{c}\text { Saturated Fat } \\
\text { Trans Fat } \\
\text { Polyunsaturated } \\
\text { Fat } \\
\text { Monounsaturated } \\
\text { Fat } \\
\text { Levitation Height } \\
\text { (mm) }\end{array}$} & 1 & 13 & 2 & 2 & 2 & 2 \\
\hline & 0 & 0 & 0 & 0 & 0 & 0 \\
\hline & 4 & 0.5 & 8 & 2 & 2 & 8 \\
\hline & 9 & 0.5 & 4 & 10 & 10 & 3 \\
\hline & 0.52 & 0.44 & 0.46 & 0.64 & 0.63 & 0.44 \\
\hline
\end{tabular}

\subsection{Ethanol Samples}

Ethanol samples were levitated in a $15 \mathrm{mM}$ solution of gadolinium acetylacetonate (a hydrophobic chelate of gadolinium) dissolved in 2-fluorotoluene with heat. 150 $\mu \mathrm{L}$ of ethanol-water solution was added drop-wise to the paramagnetic solution and vortexed for 5 seconds to 
decrease the droplet size. Levitation was imaged within 5 minutes of mixing the sample with the paramagnetic solution in order to minimize extraction of ethanol into the surrounding solution, to prevent droplet aggregation, and to maintain rounded droplets.

\section{Results and Discussion}

\subsection{Sample Levitation}

The apparatus (shown in Figure 1 a-d) [16] includes a compact magnetic levitation unit which levitates a sample suspended in a paramagnetic solution. Sample droplets are formed in a paramagnetic solution in a disposable glass microcapillary (depicted in Figure 1 e) by vortexing to form small droplets on the order of up to a few hundred micrometres. Oil droplets are levitated in an aqueous solution of gadolinium and ethanol-water droplets are suspended in an organic solvent with a hydrophobic chelate of gadolinium. The microcapillary inserted into a magnetic field formed by two permanent $\mathrm{NdFeB}$ magnets. The levitating sample is illuminated by a battery-powered LED through a diffuser and the image is magnified by an aspheric lens (Figure $1 \mathrm{f}-\mathrm{g}$ ). Images are captured by the built-in smartphone camera (representative images shown in Figure $1 \mathrm{~h}$ ). The images are then analysed on the same smartphone using a custom-developed Android application to output the density of the sample.

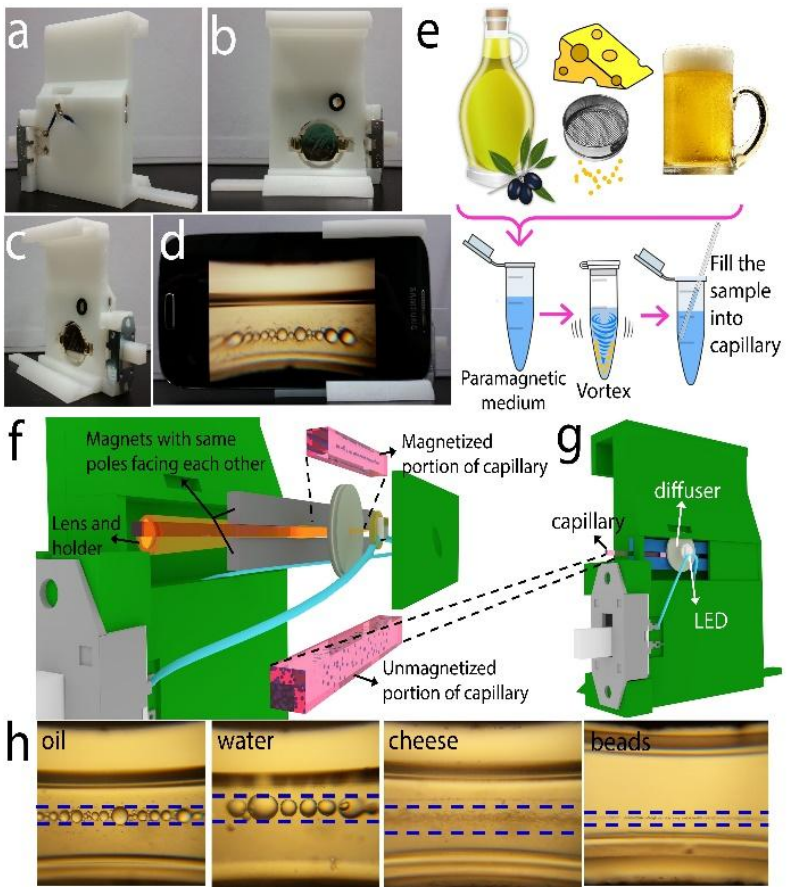

Figure 1. Magnetic levitation attachment. a) Front, b) back, and c) side views of the 3-D printed cell phone attachment. d) Samsung Galaxy S4 smartphone in the apparatus while capturing a levitation image of the sample in the microcapillary tube shown. e) Methods for levitating different food types. f-g) The magnetic levitation component of the device showing the light path from the LED, through a diffuser, through the sample, and received by the camera detector. The sample is located between the two magnets with same poles facing each other, causing alignment of the particles in the magnetized portion of the capillary. h) Images of levitation of hydrophobic oil, water, cheese particles, and polystyrene beads captured with the device.

\subsection{Image Analysis}

This analysis platform is supported with a customdeveloped Android application, Food Tester, running on the Samsung Galaxy S4 smartphone (Figure 2a). The application is developed with Android Studio, the official Android integrated development environment. It has the capability of accepting a username and password for added security (Figure $2 b$ ). The analysis comprises two main components:

1. Scanning pixels along the x-axis, averaging the pixel intensities, and saving them in an array with a length value indicating the $y$-axis coordinate.

2. Scanning pixels along the $\mathrm{x}$-axis, finding the gradients of pixel intensity changes in $\mathrm{x}$ and $\mathrm{y}$ directions, and saving them with a length value indicating the y-axis coordinate.

With the sample images in this study, the first array values peak in three general regions: (Region 1) the region between the upper edge of the microcapillary and the top edge of the image, (Region 2) the region between the lower edge of the microcapillary and the bottom edge of the image, and (Region 3) the region of confinement in which the particles are equilibrated. From this array, the gradient of pixel intensity at a y-axis location is successively compared with the five neighbouring pixels towards the top until the top image is encountered or pixel intensity falls below $5 \%$ of the maximum pixel intensity in the image - this location determines the upper edge of the microcapillary. Similarly, the lower edge is determined (blue line in Figure $2 \mathrm{c}-\mathrm{h}$ ). The magnet boundary towards the top of the image (henceforth referred to as the 'top magnet') serves as the reference point for calculating the levitation height of the particles of interest. It should be noted that captured images are flipped vertically, so 'top magnet' in the image analysis is the bottom magnet in the magnetic levitation setup. Therefore, all levitation heights are measured from the bottom magnet.

The second array values again peak at again three general regions: (Region A) top and (Region B) bottom edges of the microcapillary and (Region $C$ ) the region of confinement in which sample particles are equilibrated. Region 3 and Region C generally overlap. Based on the pixel intensities in Region 3 and the gradient values in Region $\mathrm{C}$, the region of equilibration is identified. A Gaussian distribution is fitted to the pixel intensity versus $y$-axis location data and the mean of this distribution is reported as the levitation height in pixels (seen as the black histogram with the red Gaussian fit line in Figure 2 $\mathrm{c}-\mathrm{h})$. The same technique is used to identify the edges of the magnets as is done to identify the microcapillary 
boundaries. The magnet boundaries are calculated by iteratively comparing the gradient of pixel intensity in the $y$-direction of one pixel with that of the neighboring five pixels to identify the microcapillary edges (red line in Figure $2 \mathrm{c}-\mathrm{h}$ ). The width between these edges is used to calculate the microcapillary width. This measured distance in pixels is equivalent to the inner diameter of the microcapillary, $0.7 \mathrm{~mm}$. Based on this measurement and the number of pixels between the edges of the microcapillary, a conversion factor is obtained to convert the levitation height from pixels to millimeters.

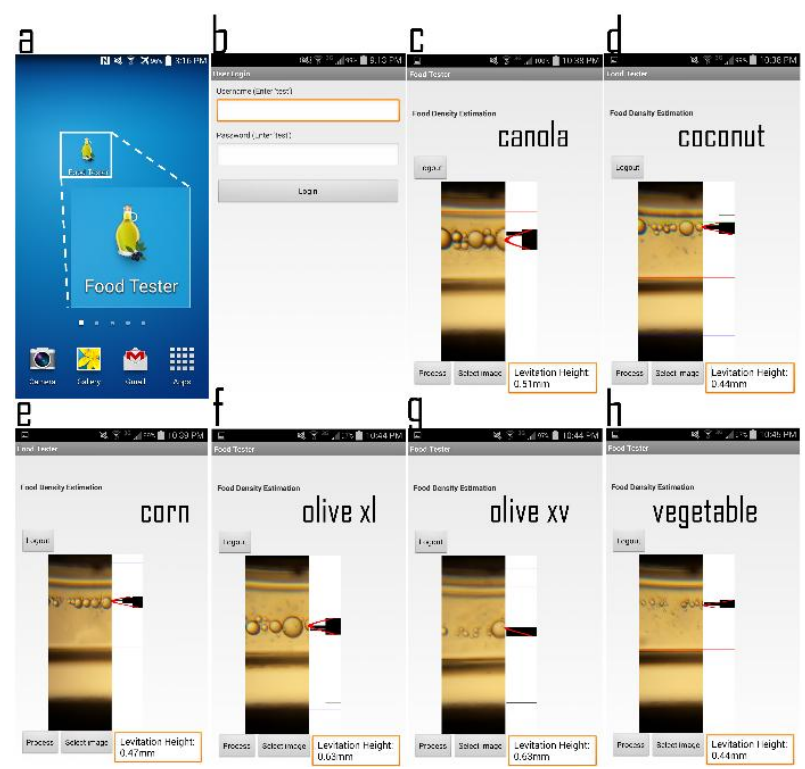

Figure 2. Demonstration of the Food Tester automated levitation height analysis application. a) The icon shown on a Samsung Galaxy S4. b) The login screen to provide security. Levitation height analysis for: c) canola, d) coconut, e) corn, f) extra light olive oil, g) extra virgin olive oil, and h) vegetable oil; each image shows detection of a Gaussian fit to the levitated droplets, detection of the magnet inner edges (blue lines) and detection of the capillary inner edges (red lines), and a readout showing the levitation height in millimeters.

\subsection{Correlation between Fat Content in Oil and Levitation Height}

Figure $3 \mathrm{a}$ shows how the fat content of several oil samples is correlated to the levitation height. All samples had $14 \mathrm{~g}$ total fat (100\% of the serving size), but varying compositions of saturated fat, monounsaturated fat, and polyunsaturated fat (all samples contained $0 \mathrm{~g}$ trans-fat) The saturated fat content is similar in all samples, so no conclusions may be drawn directly from this data alone. Based on the experimental data, the levitation height decreases as the polyunsaturated fat content increases with a good coefficient of determination $\left(\mathrm{R}^{2}=0.93\right)$. In addition, the levitation height increases with as the monounsaturated fat content increases. This trend is approximately linear, but has a low coefficient of determination $\left(\mathrm{R}^{2}=0.84\right)$. However, when adding the monounsaturated fat content (which only has one carbon- carbon double bond, compared to multiple double bonds in polyunsaturated fat) with the saturated fat content (which has no double bonds), the trend has a much improved linear correlation $\left(\mathrm{R}^{2}=0.92\right)$. This improvement in the linear trend when combining these two types of fats implies that their effects on the levitation height are similar, so this method is less sensitive to the difference between the monounsaturated fat and saturated fat content than it is to the polyunsaturated fat content. Based on the linear trend given, it is possible to estimate (1) the total saturated and monounsaturated fat content of any given oil sample and (2) the polyunsaturated fat content of that sample based on levitation height with this platform.

It should be noted that coconut oil was also tested, but excluded from these linear fit correlations. This sample exists in the solid state at room temperature. The levitation height of $0.44 \mathrm{~mm}$ would predict this sample to have high saturated and monounsaturated fat content and low polyunsaturated fat content according to the trends established with liquid samples. But in fact, coconut oil is reported to have $97 \%$ saturated and monounsaturated fats and $4 \%$ polyunsaturated fats, which is opposite of the trends seen in the liquid samples. We hypothesize that this inconsistency is due to an increase in density associated with the solid phase of coconut oil compared to the liquid phase in other liquid phase oils, causing this sample to levitate at a lower height.

These results demonstrate the ability to observe physical properties of oil through the levitation height of sample droplets with our platform. However, these same methods with aqueous solutions of gadolinium as the paramagnetic medium may be used to levitate any hydrophobic sample.

\subsection{Correlation between Alcohol Content and Levitation Height}

Figure $3 \mathrm{~b}$ demonstrates a positive correlation between the alcohol (ethanol) content in a water solution with the levitation height of droplets. The correlation is approximately linear $\left(\mathrm{R}^{2}=0.98\right)$. The range of concentration detection under these conditions is limited to $20 \%$ due to the width of the capillary - higher concentrations levitate at the upper limit of the microcapillary. Greater concentrations may be detected by decreasing the density of the suspending medium to shift all levitation heights downward (by decreasing the buoyancy force acting of the droplets) or by increasing the concentration of gadolinium in the paramagnetic medium (by increasing the magnetic force on the droplets toward the centerline.)

These results demonstrate the ability to determine the concentration of a solute in an aqueous solvent using this platform and a solution of gadolinium chelate which is soluble in the organic solvent used. This method is extendable to any solute which increases the mass of the solution but does not cause significant volume change upon dissolution. 

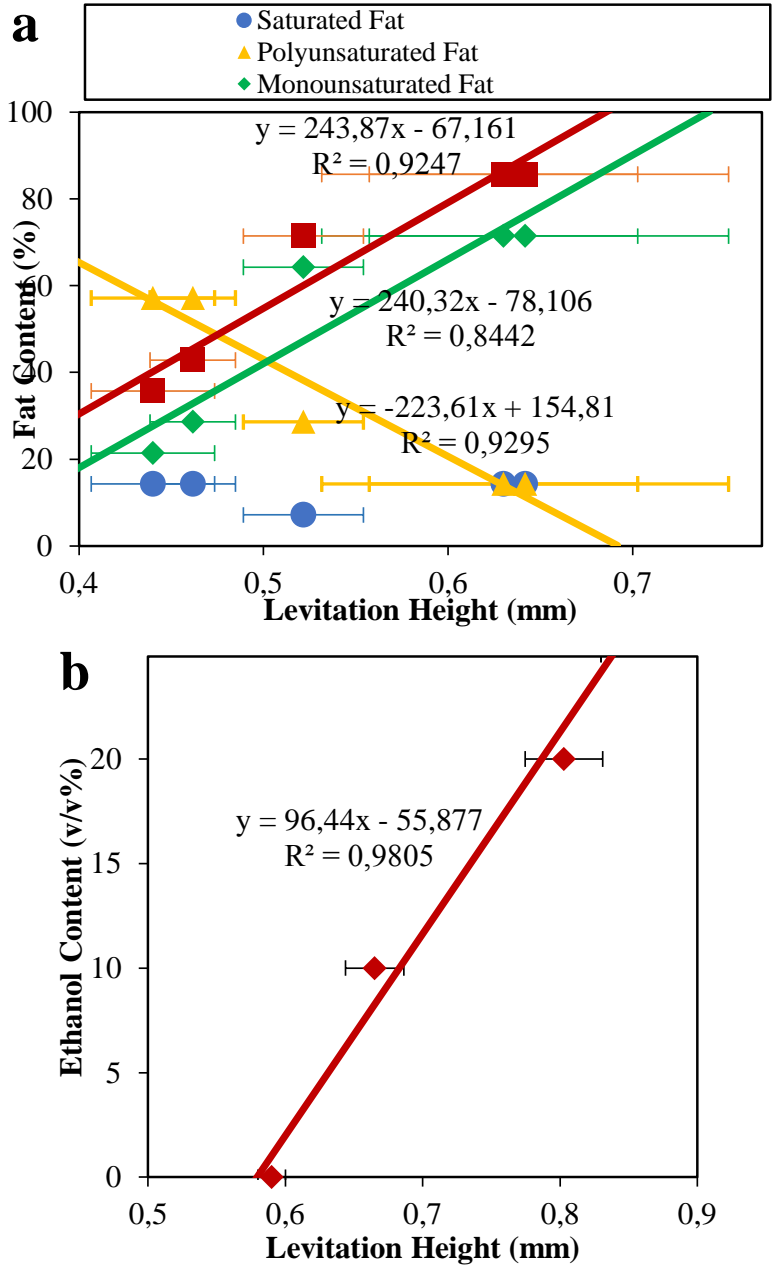

Figure 3: a) Correlation between fat content (including saturated fat, monounsaturated fat, and polyunsaturated fat) and levitation height with linear trend lines. b) Correlation between alcohol content (v/v\%): 0\%, 10\%, and $20 \%$, and levitation height with a linear trend line.

\section{Conclusion}

We demonstrated a digital and lightweight food testing platform, which employs permanent magnets with the same poles are facing each other, disposable microcapillary, optical components (aspheric lens, diffuser, LED and printed lens frame), and a customdeveloped Android application running on the same smart-phone.

Here, we have demonstrated applicability to oil and alcohol solution density-based analysis. However, the device's density measurement functions may easily be extended to any hydrophobic liquid or any aqueous solutions using similar sample preparation and analytical methods. It may also be extended to solid food products, provided that the samples may be reduced to a micronscale size around $10-100 \mu \mathrm{m}$ in diameter. As the magnetic levitation utilized here depends on the density of food particles/droplets (and not the volume), any irregularity in shape and/or volume of these food particles does not interfere with the feasibility of this approach. Therefore, this allows simple, inexpensive, and fast preparation of these food particles (e.g. with a simple sieve) without needing any elegant device. It should also be noted that the paramagnetic solutions used here are limited by the range of density liquids they can detect. However, it has been shown that the range can be shifted by changing the density of the paramagnetic medium and it can be expanded by increasing the concentration of the paramagnetic medium [16-20].

The apparatus and testing methods presented here are readily applicable to the food production industry, where there is demonstrated need to promptly and accurately perform density-based analysis of a wide range of samples for human consumption. This device provides a portable and inexpensive alternative to alternative devices available on the market. Based on the low cost and compatibility with standard smartphones, this platform is also applicable to home-based usage, such as for monitoring the alcohol content of a batch of an alcoholic beverage in a home brewery. Digital analysis reduces the risk of user error and differences between multiple users, improving overall repeatability in the field. This platform also offers the potential to track multiple sample results over time using the build-in application in order to detect statistically significant deviations from normal in any of the readings taken; such analysis can also be done natively on the smartphone without a need for an additional PC.

\section{Acknowledgement}

We would like to thank Chu Hsiang-Yu for his artful representation of the attachment in Figure 1f-g. ST acknowledges the University of Connecticut Research Excellence Program award for financial support of this research. SK acknowledges the support of the National Science Foundation Graduate Research Fellowship (DGE-1247393).

\section{Author's Contributions}

ST designed the experiments. SK performed the experiments and analyzed the results. ST and SK wrote the manuscript.

\section{Ethics}

There are no ethical issues after the publication of this manuscript.

\section{References}

1. Executive Policy Council. WFP Food Safety and Quality Management System. Eleventh Meeting of the Executive Policy Council, 2010.

2. Pomeranz, Y, Meloan, CE. Food Analysis Theory And Practice; Springer Press: United States, 1994.

3. Mirica, KA, Phillips, ST, Mace, CR, Whitesides, GM. 2010 Magnetic levitation in the analysis of foods and water. Journal of Agricultural and Food Chemistry; 58(11): 6565-6569.

4. Anil-Inevi, M, Yaman, S, Yildiz, AA, Mese, G, Yalcin-Ozuysal, O, 
Tekin, HC, Ozcivici, E. 2018. Biofabrication of in situ Self Assembled 3D Cell Cultures in a Weightlessness Environment Generated using Magnetic Levitation. Scientific Reports; 8: 7239.

5. Turker, E, Arslan-Yildiz, A. 2018. Recent Advances in Magnetic Levitation: A Biological Approach from Diagnostics to Tissue Engineering. ACS Biomaterials Science and Engineering; 4(3): 787799.

6. Lockett, MR, Mirica, KA, Mace, CR, Blackledge, RD, and Whitesides, GM. 2013. Analyzing forensic evidence based on density with magnetic levitation. Journal of Forensic Science; 58(1): 40-45.

7. Atkinson, MBJ, Bwambok, DK, Chen, J, Chopade, PD, Thuo, MM, Mace, CR, Mirica, KA, Kumar, AA, Myerson, AS. 2013. Using magnetic levitation to separate mixtures of crystal polymorphs. Angewandte Chemie International Edition; 52(39): 10208-10211.

8. Tasoglu, S, Yu, CH, Gungordu, HI, Guven, S, Vural, T, Demirci, U. 2014. Guided and magnetic self-assembly of tunable magnetoceptive gels. Nature communications; 5: 4702.

9. Tasoglu, S, Yu, CH, Liaudanskaya, V, Guven, S, Migliaresi, C Demirci, U. 2015. Magnetic levitational assembly for living material fabrication. Advanced healthcare materials; 4 (10): 1469-1476.

10. Winkleman, A, Perez-Castillejos, R, Gudiksen, KL, Phillips, ST, Prentiss, M, Whitesides, GM. 2007. Density-based diamagnetic separation: devices for detecting binding events and for collecting unlabeled diamagnetic particles in paramagnetic solutions. Analytical Chemistry; 79(17): 6542-6550.

11. Shapiro, ND, Soh, S, Mirica, KA, Whitesides, GM. 2012. Magnetic levitation as a platform for competitive protein-ligand binding assays. Analytical Chemistry; 84(14): 6166-6172.

12. Subramaniam, AB, Gonidec, M, Shapiro, ND, Kresse, KM, Whitesides, GM. 2015. Metal-Amplified Density Assays, (MADAs), including a Density-Linked Immunosorbent Assay (DeLISA). Lab on a Chip; 15(4): 1009-1022.

13. Iberian Coppers S.A., Using an Alcoholmeter \& Method, https://www.copper-alembic.com/en/page/using-an-alcoholmetermethod, 2016 (accessed 4.12.2018).

14. Knowlton, S, Sencan, I, Aytar, Y, Khoory, J, Heeney, MM, Ghiran, IC, Tasoglu, S. 2015. Sickle cell detection using a smartphone. Scientific Reports; 5: 15022.

15. Knowlton, S, Joshi, A, Syrrist, P, Coskun, AF, Tasoglu, S. 2017. 3D-printed smartphone-based point of care tool for fluorescence- and magnetophoresis-based cytometry. Lab on a Chip; 17(16): 2839-2851.

16. Knowlton, S, Yu, CH, Jain, N, Ghiran, IC, Tasoglu, S. 2015. SmartPhone Based Magnetic Levitation for Measuring Densities. PLoS One; 10(8): e0134400.

17. Amin, R, Knowlton, S, Dupont, J, Bergholz, JS, Joshi, A, Hart, A, Yenilmez, B, Yu, CH, Wentworth, A, Zhao, JJ, Tasoglu, S. 2017. 3Dprinted smartphone-based device for label-free cell separation. Journal of $3 D$ printing in Medicine; 1 (3): 155-164.

18. Yenilmez, B, Knowlton, S, Tasoglu, S. 2016. Self-contained handheld magnetic platform for point of care cytometry in biological samples. Advanced Materials Technologies; 1 (9): 1600144.

19. Yenilmez, B, Knowlton, S, Yu, CH, Heeney, MM, Tasoglu, S. 2016. Label-free sickle cell disease diagnosis using a low-cost, handheld platform. Advanced Materials Technologies; 1 (5): 1600100.

20. Tasoglu, S, Khoory, JA, Tekin, HC, Thomas, C, Karnoub, AE, Ghiran, IC, Demirci U. 2015. Levitational image cytometry with temporal resolution. Advanced Materials; 27 (26): 3901-3908.

\section{SUPPLEMENTARY FILE}
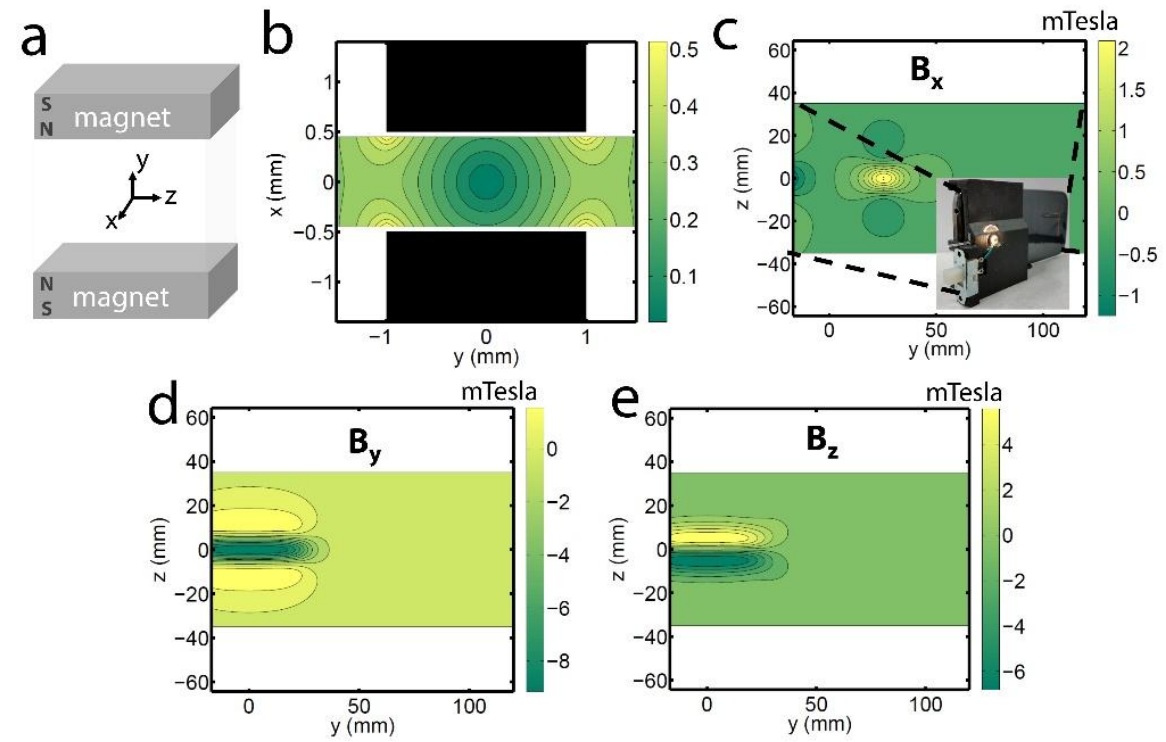

Supplementary Figure 1. Theoretical demonstration of density-based magnetic levitation of food particles. (a) Two magnets with same poles facing each other, and the coordinate system used. (b) Contour plot of magnitude of magnetic field strength at cross-sectional area (at $\mathrm{z}=0$, center of two magnets). The magnitude of the magnetic field is constrained between $0 \mathrm{~T}$ and $0.4 \mathrm{~T}$. (c-e) Magnitude of magnetic fields at the back surface of cell phone. Contour plots are given for field strengths in (c) x-direction, (d) y-direction, and (e) z-direction. 
Methods

Design and fabrication of the setup via 3D printer

The smart-phone apparatus was designed in TinkerCAD, an online application for computer aided design (Autodesk, Inc., San Rafael,

CA). The apparatus was printed with a Form 1+ high-resolution stereolithography 3D printer with black photoreactive resin (Formlabs, Somerville, MA). The main component is designed around a Samsung Galaxy S4 smart-phone (dimensions $136.6 \mathrm{~mm}$ length by $69.8 \mathrm{~mm}$ width by $7.9 \mathrm{~mm}$ depth) such that the smart-phone slides in lengthwise and is held securely upright along its length. Two N52-grade nicke plated NdFeB magnets with dimensions $50.8 \mathrm{~mm}$ length by $2 \mathrm{~mm}$ width by $5 \mathrm{~mm}$ thickness which are magnetized through the $5 \mathrm{~mm}$ thicknes (custom design, K\&J Magnetics, Inc., Pipersville, PA) are fixed with same poles facing each other $1 \mathrm{~mm}$ apart. A lens (described in a later section) is used to achieve magnification of the sample and is secured in a holder and placed between the built-in camera of the smart-phone and the sample. The sample is illuminated on the opposite side from the camera using an LED connected in series to a standard slide switch (GF-1123-0025, CW Industries, Southampton, PA) and a CR 2032 3V battery (CR2032, Panasonic, Newark, NJ) with solid hookup wire and solder. The switch is fixed to the apparatus with adhesive and the battery is held with a battery holder (BU2032SM-BT-GTR, Memory Protection Devices, Inc., Farmingdale, NY)

Imaging procedure and the optical setup:
The sample is illuminated by a white LED driven with a 3V CR2032 coin battery. A 120-grit ground glass diffuser (DG05-120, Thorlabs, Inc, Newtown, NJ) between the LED and capillary tube ensures uniformity of illumination across the imaging field of view (FOV). Objects within this FOV are imaged onto the active area of the cellphone camera (Sony IMX135, 1.12 $\mu$ m pixel pitch, 4208x3120 pixels) by using a 4-f imaging system. This 4-f imaging system is formed by using the built-in lens unit of the cellphone camera with an effective focal length (EFL) of $4.2 \mathrm{~mm}$ and an external aspheric lens with diameter $6.33 \mathrm{~mm}$, numerical aperture $0.64 \mathrm{~mm}$, and effective focal length $4.03 \mathrm{~mm}$ (87-161, Edmund Optics, Barrington, NJ). The spacing between the camera lens and the external lens is adjusted as the summation of their focal lengths $(\sim 8.23 \mathrm{~mm})$. Also, the ratio between these focal lengths provides approximately unit magnification $(\mathrm{M}=$ $4.2 / 4.03=\sim 1.04)$, and this ratio sets the effective pixel size of the imaging optics on the object plane as $\sim 1.17 \mu \mathrm{m}$. Working with this effective pixel size provides a sufficient sampling rate for reliable localization of objects and does not reduce the FOV, which spans entire depth of the capillary tube $(1 \mathrm{~mm})$ without aberrations. The alignment of the imaging system was experimentally optimized to maximize the resolution and minimize optical aberrations (Pincushion distortions, chromatic aberrations, and vignetting). Images were captured using the stock camera application that comes standard with the Samsung Galaxy $\mathrm{S} 4$. Autofocus was used to focus images by manually selecting the region of confinement.

Mathematical modeling of magnetic field distribution.

The magnetic field, B, follows:

$$
\begin{aligned}
& \mathrm{B}(\mathrm{x}, \mathrm{y}, \mathrm{z}) \\
& =\frac{\mu_{0} \mathrm{M}_{s}}{4 \pi} \sum_{\mathrm{k}=1}^{2}(-1)^{\mathrm{k}} \int_{\mathrm{y}_{1}}^{\mathrm{y}_{2}} \int_{\mathrm{x}_{1}}^{\mathrm{x}_{2}} \frac{\left[\left(\mathrm{x}-\mathrm{x}^{\prime}\right) \hat{\mathrm{x}}+\left(\mathrm{y}-\mathrm{y}^{\prime}\right) \hat{\mathrm{y}}+\left(\mathrm{z}-\mathrm{z}_{\mathrm{k}}\right) \hat{\mathrm{z}}\right] \mathrm{dx} \mathrm{x}^{\prime} d \mathrm{y}^{\prime}}{\left[\left(\mathrm{x}-\mathrm{x}^{\prime}\right)^{2}+\left(\mathrm{y}-\mathrm{y}^{\prime}\right)^{2}+\left(\mathrm{z}-\mathrm{z}_{\mathrm{k}}\right)^{2}\right]^{3 / 2}}
\end{aligned}
$$

The X-component: $\mathrm{B}_{\mathrm{x}}$ follows from Eq. (1)

$$
B_{x}(x, y, z)=\frac{\mu_{0} M_{s}}{4 \pi} \sum_{k=1}^{2}(-1)^{k} \int_{y_{1}}^{y_{2}} \int_{x_{1}}^{x_{2}} \frac{\left(x-x^{\prime}\right) d x^{\prime} d y^{\prime}}{\left[\left(x-x^{\prime}\right)^{2}+\left(y-y^{\prime}\right)^{2}+\left(z-z_{k}\right)^{2}\right]^{3 / 2}} .
$$

Integration with respect to $x^{\prime}$ gives

$$
B_{x}(x, y, z)=\frac{\mu_{0} M_{s}}{4 \pi} \sum_{k=1}^{2} \sum_{m=1}^{2}(-1)^{k+m} \int_{y_{1}}^{y_{2}} \frac{d y^{\prime}}{\left[\left(x-x_{m}\right)^{2}+\left(y-y^{\prime}\right)^{2}+\left(z-z_{k}\right)^{2}\right]^{1 / 2}} .
$$

The remaining y' integration can be evaluated by making a change of variable to $\alpha=y-y$ '. The resulting field expression is

$$
\begin{gathered}
\mathrm{B}_{\mathrm{x}}(\mathrm{x}, \mathrm{y}, \mathrm{z})=\frac{\mu_{0} \mathrm{M}_{\mathrm{s}}}{4 \pi} \sum_{\mathrm{k}=1}^{2} \sum_{\mathrm{m}=1}^{2}(-1)^{\mathrm{k}+\mathrm{m}} \ln \left[\mathrm{F}\left(\mathrm{x}, \mathrm{y}, \mathrm{z}, \mathrm{x}_{\mathrm{m}}, \mathrm{y}_{1}, \mathrm{y}_{2}, \mathrm{z}_{\mathrm{k}}\right)\right], \\
\mathrm{F}\left(\mathrm{x}, \mathrm{y}, \mathrm{z}, \mathrm{x}_{\mathrm{m}}, \mathrm{y}_{1}, \mathrm{y}_{2}, \mathrm{z}_{\mathrm{k}}\right)=\frac{\left(\mathrm{y}-\mathrm{y}_{1}\right)+\left[\left(\mathrm{x}-\mathrm{x}_{\mathrm{m}}\right)^{2}+\left(\mathrm{y}-\mathrm{y}_{1}\right)^{2}+\left(\mathrm{z}-\mathrm{z}_{\mathrm{k}}\right)^{2}\right]^{1 / 2}}{\left(\mathrm{y}-\mathrm{y}_{2}\right)+\left[\left(\mathrm{x}-\mathrm{x}_{\mathrm{m}}\right)^{2}+\left(\mathrm{y}-\mathrm{y}_{2}\right)^{2}+\left(\mathrm{z}-\mathrm{z}_{\mathrm{k}}\right)^{2}\right]^{1 / 2}}
\end{gathered}
$$

The y-component: $\mathrm{B}_{\mathrm{y}}$ also follows from Eq. (1),

$$
B_{y}(x, y, z)=\frac{\mu_{0} M_{s}}{4 \pi} \sum_{k=1}^{2}(-1)^{k} \int_{y_{1}}^{y_{2}} \int_{x_{1}}^{x_{2}} \frac{\left(y-y^{\prime}\right) d x^{\prime} d y^{\prime}}{\left[\left(x-x^{\prime}\right)^{2}+\left(y-y^{\prime}\right)^{2}+\left(z-z_{k}\right)^{2}\right]^{3 / 2}} .
$$

Integration with respect to y' gives

$$
\mathrm{B}_{\mathrm{y}}(\mathrm{x}, \mathrm{y}, \mathrm{z})=\frac{\mu_{0} \mathrm{M}_{\mathrm{s}}}{4 \pi} \sum_{\mathrm{k}=1}^{2} \sum_{\mathrm{m}=1}^{2}(-1)^{\mathrm{k}+\mathrm{m}} \int_{\mathrm{x}_{1}}^{\mathrm{x}_{2}} \frac{\mathrm{dx}^{\prime}}{\left[\left(\mathrm{x}-\mathrm{x}^{\prime}\right)^{2}+\left(\mathrm{y}-\mathrm{y}_{\mathrm{m}}\right)^{2}+\left(\mathrm{z}-\mathrm{z}_{\mathrm{k}}\right)^{2}\right]^{1 / 2}}
$$

The remaining $x^{\prime}$ integration is evaluated using a change of variable $\alpha=x-x^{\prime}$. The resulting field expression is

$$
\begin{gathered}
B_{y}(x, y, z)=\frac{\mu_{0} M_{s}}{4 \pi} \sum_{k=1}^{2} \sum_{m=1}^{2}(-1)^{k+m} \ln \left[H\left(x, y, z, x_{1}, x_{2}, y_{m}, z_{k}\right)\right] . \\
H\left(x, y, z, x_{1}, x_{2}, y_{m}, z_{k}\right)=\frac{\left(x-x_{1}\right)+\left[\left(x-x_{1}\right)^{2}+\left(y-y_{m}\right)^{2}+\left(z-z_{k}\right)^{2}\right]^{1 / 2}}{\left(x-x_{2}\right)+\left[\left(x-x_{2}\right)^{2}+\left(y-y_{m}\right)^{2}+\left(z-z_{k}\right)^{2}\right]^{1 / 2}} .
\end{gathered}
$$

The z-component: $B_{z}$ is given by 


$$
B_{z}(x, y, z)=\frac{\mu_{0} M_{s}}{4 \pi} \sum_{k=1}^{2}(-1)^{k} \int_{y_{1}}^{y_{2}} \int_{x_{1}}^{x_{2}} \frac{\left(z-z_{k}\right) d x^{\prime} d y^{\prime}}{\left[\left(x-x^{\prime}\right)^{2}+\left(y-y^{\prime}\right)^{2}+\left(z-z_{k}\right)^{2}\right]^{3 / 2}} .
$$

The $\mathrm{x}^{\prime}$ integration is performed using a change of variable $\alpha=\mathrm{x}-\mathrm{x}$ '

$$
\begin{gathered}
B_{z}(x, y, z)=\frac{\mu_{0} M_{s}}{4 \pi} \int_{y_{1}}^{y_{2}} \int_{x-x_{1}}^{x-x_{2}} \frac{z-z_{k}}{\left[\alpha^{2}+\left(y-y^{\prime}\right)^{2}+\left(z-z_{k}\right)^{2}\right]^{3 / 2}} d \alpha d y^{\prime} \\
=\frac{\mu_{0} M_{s}}{4 \pi} \sum_{k=1}^{2} \sum_{n=1}^{2}(-1)^{k+n+1}\left(z-z_{k}\right)\left(x-x_{n}\right) \int_{y_{1}}^{y_{2}} \frac{d y^{\prime}}{\left[\left(y-y^{\prime}\right)^{2}+\left(z-z_{k}\right)^{2}\right] \sqrt{\left(y-y^{\prime}\right)^{2}+b^{2}}},
\end{gathered}
$$

where $b^{2}=\left(x-x_{n}\right) 2+\left(z-z_{k}\right)^{2}$. The remaining $y^{\prime}$ integration is performed using a change of variable $\gamma=y-y^{\prime}$. This gives

$$
\mathrm{B}_{\mathrm{z}}(\mathrm{x}, \mathrm{y}, \mathrm{z})=\frac{\mu_{0} \mathrm{M}_{\mathrm{s}}}{4 \pi} \sum_{\mathrm{k}=1}^{2} \sum_{\mathrm{n}=1}^{2} \sum_{\mathrm{m}=1}^{2}(-1)^{\mathrm{k}+\mathrm{n}+\mathrm{m}} \tan ^{-1}\left[\frac{\left(\mathrm{x}-\mathrm{x}_{\mathrm{n}}\right)\left(\mathrm{y}-\mathrm{y}_{\mathrm{m}}\right)}{\left(\mathrm{z}-\mathrm{z}_{\mathrm{k}}\right)} \mathrm{g}\left(\mathrm{x}, \mathrm{y}, \mathrm{z} ; \mathrm{x}_{\mathrm{n}}, \mathrm{y}_{\mathrm{m}}, \mathrm{z}_{\mathrm{k}}\right)\right],
$$

Here, we solved equations 4, 7, and 10 to plot magnetic field distribution (Supplementary Figure 1). Food particles will be driven towards regions of minimal magnetic flux density (along the symmetry line, i.e. centerline between two magnets) and can be spatially confined in 3D or 2D magnetic traps. We plotted magnetic field distribution at the back surface of cell p hone (Supplementary Figure 1c-e). 